\title{
Environmental Monitoring using Autonomous Vehicles: A Survey of Recent Searching Techniques
}

\author{
Behzad Bayat $^{\mathrm{a}}$, Naveena Crasta ${ }^{\mathrm{b}}$, Alessandro Crespi $^{\mathrm{a}}$, António M. Pascoal ${ }^{\mathrm{b}}$, Auke Ijspeert $^{\mathrm{a}}$ \\ ${ }^{a}$ Biorobotics laboratory (BioRob), Ecole polytechnique fédérale de Lausanne (EPFL), 1015 Lausanne, Switzerland \\ ${ }^{b}$ Institute for Systems and Robotics (ISR), Instituto Superior Técnico (IST), University of Lisbon \\ 1049-001 Lisboa, Portugal
}

\begin{abstract}
Autonomous vehicles are becoming an essential tool in a wide range of environmental applications that include ambient data acquisition, remote sensing, and mapping of the spatial extent of pollutant spills. Among these applications, pollution source localization has drawn increasing interest due to its scientific and commercial interest and the emergence of a new breed of robotic vehicles capable of performing demanding tasks in harsh environments without human supervision. In this task, the aim is to find the location of a region that is the source of a given substance of interest (e.g. a chemical pollutant at sea or a gas leakage in air) using a group of cooperative autonomous vehicles. Motivated by fast paced advances in this challenging area, this paper surveys recent advances in searching techniques that are at the core of environmental monitoring strategies using autonomous vehicles.
\end{abstract}

Keywords: Autonomous vehicles, Environmental monitoring, Pollution detection, Odor tracking

\section{Introduction}

Autonomous vehicles, such as flying, marine, and terrestrial robots, have made significant inroads into the area of source localization in the context of explosive and drug detection, sensing of leaking or hazardous chemicals, pollution monitoring and, in the specific case of the marine environment, hydrothermal vent source localization, plume tracing, and heat source seeking. Among other, localization of toxic pollutant sources is an important as well as a challenging issue in many environment related areas. In order to reduce the impact of pollutants on the environment, it is imperative to localize the sources so as to devise an effective control strategy.

This problem has been approached in two distinct manners. The first approach consists of a fixed network of sensors that collect and exchange measurements in order to estimate the location

\footnotetext{
This work was supported by Envirobot, a project of the Swiss NanoTera program, Natural Sciences. The work of N. Crasta was supported by the European Commission under the H2020-ICT-2014 WiMUST Project (Grant Agreement No. 645141).

Email addresses: behzad bayat@epfl.ch (Behzad Bayat), ncrasta@isr.ist.utl.pt (Naveena Crasta), alessandro.crespi@epfl.ch (Alessandro Crespi), antonio@isr.ist.utl.pt (António M. Pascoal), auke.ijspeert@epfl.ch (Auke Ijspeert)
} 
of the source. This localization scheme requires the use of reference nodes (nodes with known locations), thus implying expensive and time-consuming deployment, calibration, and recovery operations. For example, in the case of underwater applications, the nodes must be deployed at the surface and/or on the seabed, with consequent escalating costs. Furthermore, this strategy severely limits the coverage area, an undesirable feature that can only be overcome by the approach described next.

The second approach involves the use of groups of vehicles equipped with appropriate sensor suites, that move collectively towards the source location in a cooperative manner. This approach is more dynamic, flexible, and suitable for multiple source localization, but requires resolving many challenging technical problems such as, endurance, planning, coordination, communication, cooperation, and navigation of all the vehicles In this context, cooperative localization, with the objective of reducing operational costs, there has been increasing interest in the development of methods for cooperative source localization using vehicles that have limited or no position information, thus dispensing with the need for expensive navigation systems.

This area of research is vast and defies a simple summary. Hence, in this article we focus on the key developments witnessed in the last five years. The reader is referred to [1] and the references therein for earlier developments in the area, with a focus on advancements and applications of robots for environmental monitoring over the period of twenty years preceding the abovementioned publication. The presentation in [1] includes sections on marine and atmospheric plume detection and localization, as well as adaptive sampling. Interesting references in the area of biologically-inspired search algorithms can be found in [2], which surveys some of the strategies used, from single cells to higher level organisms and animals, as a means to answer extremely interesting questions in the fields of animal behavior, ecology, and evolution. A detailed survey on odor tracing methods and a review of autonomous underwater vehicle (AUV) autonomy and data-driven sample strategies can be found in $[3,4]$. Sensors are obviously an essential part of the source localization problem. However, sensing methods and platforms are a very broad field that is out of the scope of this paper. The interested reader is referred to the review articles $[5,6]$ for sensing systems in air or water.

The organization of the paper is as follows. In Section 2 we discuss various experimental setups that include different robotic platforms. Section 3 provides a comprehensive discussion on recent source localization techniques developed in the last half a decade using single and multiagents. Finally, in Section 4 we summarize our findings and draw attention to some future directions.

\section{Robotic Platforms and Field Experiments}

From a practical standpoint, the success of autonomous search methods hinges upon their performance in field experiments, which depends strongly on the platforms used and the operating conditions encountered. The choice of platforms depends on a number of key issues. In what follows we briefly present recent advances in this topic, with a strong emphasis on marine robotic platforms.

Source localization missions are lengthy and demand good maneuverabilities in order to explore a large area. Thus, in this context, endurance and maneuverability are the two decisive 
factors in the selection of a robotic platform. Furthermore, because most pollution measurements are taken at discrete instants of time and the sensors used require some latency time to detect particles in air or water, the robots must have the capability of station keeping. Ground and marine vehicles have been the two types of robots of choice used in the past half a decade. In the early stages, there have been attempts to use underground burrowing robots for underground gas leakage tracing [7], quadcopters for plume tracing [8], and unmanned aerial vehicles (UAVs) for microbe detection [9]. Unfortunately, the disturbance effect of drones on sensor measurements and their short endurance have severely limited their application. In what concerns ground operations, most robotic platforms are wheeled and in particular nonholonomic unicycle-modeled robots (two wheel differential drive with additional passive wheels) are popular, due to their simple design and superior maneuverability $\left[10^{\bullet}\right],[11,12,13,14],\left[15^{\bullet}\right]$.

Among all types of vehicles, marine robots are probably the most challenging to build and operate in the adverse conditions imposed by the water medium. Factors such as cost, ease of deployment and recovery, energy limitations, and safety, have limited contemporary AUVs to relatively small sizes (few meters). Current AUVs are typically powered by propellers of low efficiency, which are driven by electric motors. The low efficiency along with the poor transient response of the propellers have led to the low endurance of AUVs. A comparative study on the use of underwater gliders, AUVs, and remotely operated vehicles (ROVs) for plume detection and tracking $\left[16^{\circ}\right]$, concludes that actively propelled AUVs are the most well suited platform for such tasks due to their autonomy and sufficient payloads, despite their shorter endurance. The results of field tests with AUVs for source tracing purposes can be found in $[17,18]$. In this context, it is also worth mentioning other platform such as the combination of Lagrangian drifters (an oceanographic floating device) and AUVs that are used in the context of environment monitoring, which is indirectly related to plume tracing. The authors in [19] have presented a combination of a Lagrangian drifter and an AUV to map an area around it, if the drifter detects a patch of desired substance.

To overcome their low efficiency and eliminate the disturbances caused by the propellers, there is a new breed of autonomous bio-inspired marine robots, which do not use propellers for vehicle thrusting. The robotic crayfish with two arms that mimics the maxillipeds of a crayfish [20], swimming eel-like robot that uses body undulations for maneuvering [21], and robotic fishes that use caudal swimming to provide forward thrust $[22,23]$ are few examples of bio-inspired robotic platforms. In [20], the authors used the robotic crayfish for chemical source localization purposes in a small controlled aquatic environment. The robot uses its wheels to move at the bottom of a water reservoir and uses its two arms to improve the detection of chemical substances in water. The robotic fish developed in [22] is used for the applications of aquatic sampling and aquatic profiling of chemical diffusion reported in [24] and [25], respectively. The swimming eel-like robot [21] is designed to be used for pollution localization [ $26^{\bullet \bullet}$ ] with little disturbance of chemical substance distribution in water, while in [23] the authors use the robotic fish for extremum seeking.

Setting aside the platforms, the variability of operating conditions in a field experiment may lead to poor performance in the presence of external disturbances not taken explicitly into account during the design phase. Notice that experimental results such as odor tracing in a wind tunnel [10 •], [11, 12, 13, 14], [15 $]$, underground gas leakage tracing in a and pool [7], and source tracing in a controlled water reservoir $[20,23]$, were all carried out in controlled environments. On the other 


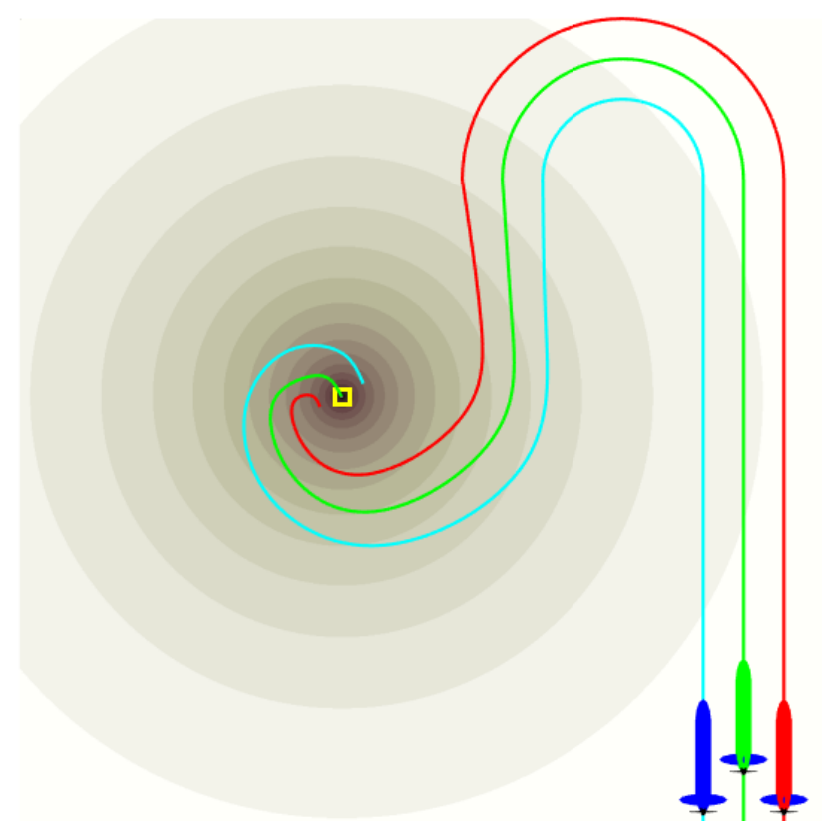

Figure 1 (adapted from [29]): Example of chemotaxis of three agents using a leader-follower approach. The brown heat map represents expected pollution level at each location. The red, blue, and green tracks indicate movement of corresponding agents in a group formation. The agents start from bottom right without any knowledge about the source location. The strategy starts with a lawn mower sweeping trajectory. Whenever some intensity of the source signal (e.g. pollution level) is detected, the agents will follow the gradient towards the source indicated by the yellow square.

hand, the underwater plume tracing reported in $[17,18]$ was carried out in open waters.

\section{Source Localization Methods}

Some of the methods for source localization can be traced back to those described in [27], involving the use of classic search patterns (e.g. parallel line, creeping line, square, sector, and barrier patrol search patterns) inspired by the techniques used in the allied search for U-boats in the Bay of Biscay during World War II. From an historical perspective, an interesting survey of early search theory and applications can be found in [28], where the authors addressed a number of practical problems in the areas of search and rescue, mineral exploration, surveillance, and fishing. Each of these applications can be (in)directly related to environmental monitoring. In the following subsections, we present a categorized but concise review of recent autonomous search methods for environmental monitoring and/or pollution source detection.

\subsection{Gradient-based (Chemotaxis) method}

Intuitively, to trace a chemical plume or localize a pollutant source, one of the desirable features of any tracing or localizing algorithm is the ability to sense the concentration of a particular chemical. This is precisely the concept of Chemotaxis, first reported in 1881, which refers to the movement of an organism caused by a chemical stimulus [30]. In simple words, chemotaxis 
consists of following the local gradient of a chemical concentration. In this context plume concentration is viewed as a scalar map with a maximum at the source location and chemotaxis yields a procedure to solve a particular optimization problem. Since its inception, due to its simplicity, chemotaxis has been exploited by many researchers to develop source seeking strategies for single or multiple agents.

We start our review with the single agent framework. In [8], a pseudo gradient-based method for the application of gas source localization was reported and validated in an open air field with the aid of a quadcopter. Inspired by [31], the vehicle tries to find the plume by sweeping crosswind and then switching to the pseudo gradient-based method by comparing the odor concentration with its previous position and directing towards the larger concentration region.

Comparative studies reported in [13] and [7] described experimental results of different chemotaxis algorithms in an in-air and underground source seeking scenarios, respectively. In both scenarios, the algorithm inspired by Escherichia coli movement resulted in variable and inefficient source seeking performance, due to its nature of random change of direction of movement and sensitivity to added measurement noise. In [13], algorithms inspired by the motion of silkworm moths and dung beetles achieved a better success rate compared to a purely gradient-based method, but at the expense of traveling for a longer distance. In [7], the presented Hex-path algorithm (discretizing the search area by hexagonal cells and move along edges) outperformed the bio-inspired planarian (changing direction of movement based on consecutive measured concentrations) in a scenario of underground chemical leak localization using a burrowing robot.

An interesting approach to three dimensional (3D) environmental extremum seeking navigation of a mobile robot is described in [32]. This study is complemented by experiments using a wheeled ground robot for a smooth scalar field, reported in [33]. The strategy proposed for extremum seeking does not require the direct computation of gradients. However, it is not evident from simulations or experiments if the proposed method can be applied to the localization of a pollution source with a diffusion process in a turbulent medium, e.g. pollution sources modeled in $\left[26^{\bullet \bullet}\right]$ and $\left[34^{\bullet \bullet}\right]$. In spite of the progress done on the use of single agents for source localization, the latter have a number of limitations. For this reason, there has been a flurry of activity on the use of multiple agents, as explained in the sequel.

Moving to a multi-agent framework, there are two possibilities; fixed and flexible formations of group of agents. In [35] the authors proposed a fixed formation of mobile sensor platform for in-air chemical plume tracing in the presence of a time-varying airflow environment. For a given formation speed, the desired formation heading is given by a function of the estimated plume direction. The algorithm was validated through simulations in a large-scale advectiondiffusion airflow environment with an achieved success rate of 80\%. In [36], the authors proposed a plume tracking and localization algorithm in a turbulent flow using a fleet of agents. The key idea of this algorithm was to transform the detected turbulent flow field into a smooth scalar field in order to make the gradient well defined. The transformation is derived by thresholding the high concentration scalar field values and considering the occurrence of high concentrations that is a function of distance to the source. The derived control law was such that the direction of the movement of the agents converges to the gradient direction of the smooth scalar field, thereby moving towards the source. However, the required sampling interval is high and choosing smaller sampling intervals leads to longer or unsuccessful source tracing. Further, the results were 


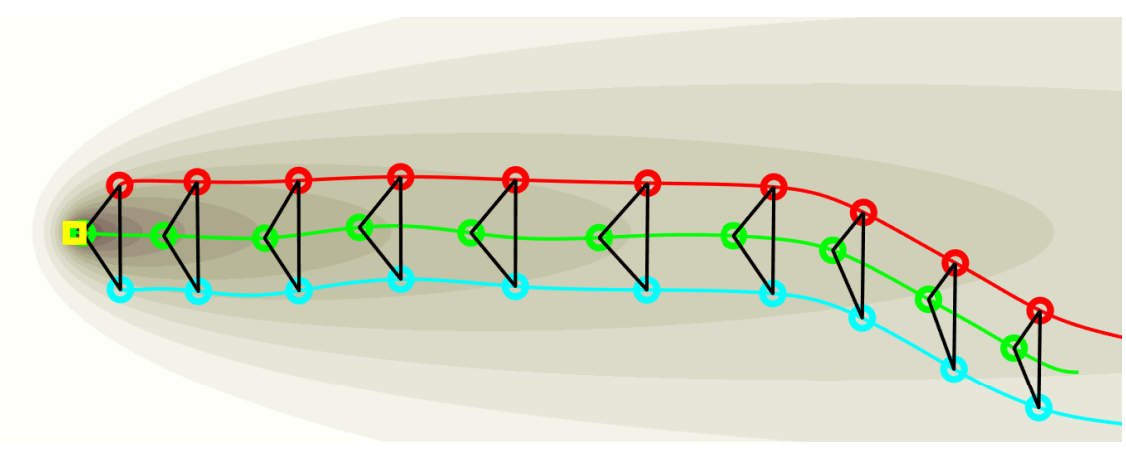

Figure 2 (adapted from [10 $\left.{ }^{\bullet}\right]$ ): Example of source localization and tracing with a triangular formation of three agents against the wind direction that is from left to right. The brown heat map represents expected pollution level at each location. Agents start from the bottom right without any knowledge of the source location. The green, red, and blue tracks represent movements of the agents, and the corresponding circles show a time lapse of their location as the mission unfolds. Black lines represent the formation of the agents at each time lapse, and the black yellow shows the location of the source.

demonstrated through simulations only.

In order to avoid the local maxima issues of gradient algorithms, [37] proposed an adaptive searching scheme called theseus gradient search (TGS) for localizing an unknown radio transmitter in indoor environments using received signal strength (RSS). The strategy combines the gradient of the RSS and exploration of regions that are not traveled in order to ensure that the robot will not get stuck in the local maxima.

A simulated leader-follower approach was presented in [29] for source localization using autonomous surface vehicles (ASVs) and AUVs based on gradient information from a scalar field of interest (see figure 1). The control law that is applied to the agents is determined by an approximation to the gradient of the particular scalar field of interest obtained by collecting measurements taken by the agents. The strategy starts with a predefined search pattern, for instance, a lawn mower sweeping trajectory. Whenever the measured intensity of the scalar field is above a certain threshold, the agents will follow the gradient towards the source.

\subsection{Wind Detection (Anemotaxis)}

Anemotaxis is the response of an organism to wind. Many insects show a positive anemotactic response (turning or flying into the wind) upon exposure to an airborne stimulus cue from a food source. In simple words, anemotaxis-driven agents focus on the advection portion of the flow. The agents navigate upstream within the plume by measuring the direction of the fluid's velocity. Moths are fascinating examples of anemotaxis agents. As early as 1983, a review of zigzagging and casting as a programmed response to follow wind-borne odor was presented [38]. Later, a study [39] investigated the search strategies of male moths to find female ones by following their scent. More specifically, the authors showed that the male moths balance counterturning and flying upwind depending on the plume structure and frequency of odor encounters, which yields to moving upwind faster and/or straighter.

Inspired by search methods taken from male moths flying upwind, similar control laws based on a behavior-based state machine have been developed on Remote Environmental Monitoring 
UnitS (REMUS) AUV [40] to trace, reacquire, and localize a plume [17]. Further on a different study [41], the authors used the same idea to install a pair of separated chemical sensors on an AUV for plume tracing in a marine simulated environment. In [42], the authors extended the moth-inspired plume tracing via a single vehicle to multiple vehicles which were validated through simulations and experimental results with a single vehicle in [18].

Recently, in [10 $\left.{ }^{\circ}\right],[11]$ a formation control scheme based on ranges from other agents was employed for odor source localization and tracing in a two dimensional (2D) wind tunnel via Laplacian feedback formation control [43] of multiple cooperative robots in a variety of formations (see figure 2). Source localization was done by plume centering of the formation based on odor measurements obtained from all the robots in the formation. With an extension to a 3D scenario, another study presents simulation and experimental results for 3D distributed plume tracking with a group of land and air robots measuring odor concentration in a wind tunnel [12]. In [44], the authors simulated a group of MEDUSA class of marine vehicles [45] measuring conductivity in a fresh water stream. These works $[12,44]$ use integration of three behaviors: upstream movement, plume centering, and Laplacian feedback formation control.

In a more recent study [46], the authors extended the source localization problem to multiple odor sources and presented localization with multiple robots in a circular formation avoiding obstacles and localizing odor sources in a simulated environment. From an optimal point of view, the formation of swarm robots that maximizes their probability of finding an odor plume in an unknown environment was studied [14]. The authors analytically showed that the optimal configuration is a diagonal upwind moving formation, facing the wind direction. This was validated through simulations and indoor experimental tests.

\subsection{Entropy Reduction (Infotaxis)}

In a turbulent medium like air or water, turbulent flow causes random and disconnected patches of odor or pollution which makes the gradient of information uncertain to be used for guidance in source seeking. Infotaxis [47], a gradient-free method, is a recent addition to the search methodology. From a theoretical stand-point, this method is based on information principles to maximize the entropy reduction. Several studies exploited this method as reported in [34 ${ }^{\bullet \bullet}$, see figure 3 , and references therein. In a different study $\left[15^{\circ}\right]$, inspired by moths, the authors attached an electroantennogram of a moth to a robot to indirectly control it. They compared four search strategies, three reactive (combination of upwind surge, spiral casting and crosswind [zigzag] casting) [48], and infotaxis [47]. They concluded that reactive searching is more efficient (yielding shorter trajectories) for higher pheromone doses while infotaxis does a better job in cases with lower doses of pheromones but with more computational cost. In [49], the authors investigated the performance of the infotaxis algorithm on a simulated 3D turbulent channel air flow for two different Reynolds numbers.

The authors in [50] have presented information theoretic search strategies based on [47] for a planar case but also extended the source localization for a group of robot(s) in a turbulent flow, in a 2D [50] and 3D [51] scenario. The clear contribution and advantage are the use of a multi-robot system which is explored in a lossless communication framework communication without any loss of information. In reality, however, underwater communications are severely affected by packet losses and delays. 




Figure 3 (adapted from [34 $\left.{ }^{\bullet}\right]$ ): Example of source localization and tracing using infotaxis method. An agent starts from the bottom right without any knowledge of the source location. The brown heat map represents expected pollution level at each location. The red track represents movements of the agent, the black circles are pollution detection, the yellow square shows the location of the source, and the green dots show hypotheses of source locations which are close to the true location. Since the current flow is to the right, the agent first sweeps cross-current and approaches the source when detections occur more often.

The infotaxis search problem on a two-dimensional grid but in a distributed form and with agents that are almost memoryless is studied in [52]. The author emphasizes that a good search strategy does not necessarily consist in always trying to approach the source, but also to move orthogonally to the estimated direction of the source, which has been shown analytically in a different application in [53]. In this strategy, which is introduced as amoebae-infotaxis model, each agent performs a random walk on the grid and when it makes a detection, it triggers a reactiondiffusion wave in the grid to attract other agents towards itself. In the long term, this will move the group towards the source. The search strategy benefits from a simple implementation for multi-agent systems, but its success is not guaranteed and it might fail in some situations.

The infotaxis approach is very promising, but as pointed out in $\left[54^{\bullet}\right]$, it only remains robust as long as the estimated parameters of the source are within a neighborhood of their true values. If the searcher agent severely underestimates or overestimates the real environment parameters, the success rate quickly drops, making infotaxis no longer feasible.

\subsection{Information Maximization (FIM)}

The concept of using a sensor model carried by one agent to decide on the best possible course of action of that agent so as to maximize the information available for localizing an underwater target has been exploited in $[53,55]$. By adopting an estimation-theoretical setting, the key idea exploited is to maximize, by a proper choice of the agent's motion, the information available for source localization. This is done by maximizing the determinant of an appropriately defined 


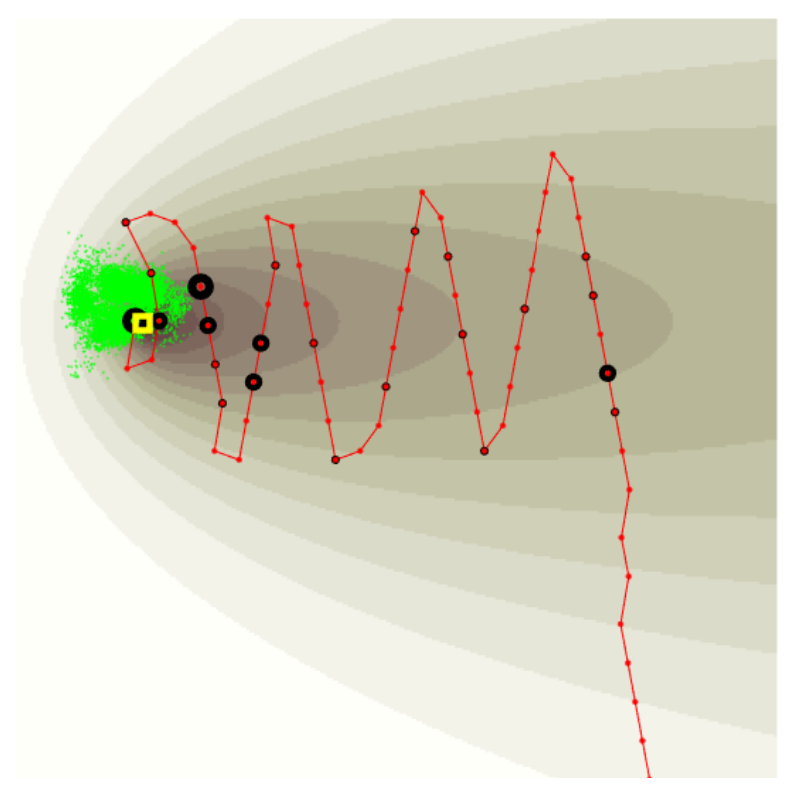

Figure 4 (adapted from $\left[26^{\bullet \bullet}\right]$ ): Example of source localization and tracing using the information maximization (FIM) method. An agent starts from bottom right without any knowledge about the source location. The red track represents movements of the agent, black circles are pollution detections (diameters proportional to pollution levels), the yellow square shows the location of the source, the green dots show hypotheses of source locations, which are close to the true location. The flow direction is to the right and the brown heat map represents the expected pollution level at each location.

Fisher information matrix (FIM), which yields a measure of the minimum covariance of the target estimation error that can possibly be achieved with any unbiased estimator. Referring to [55], the strategy is to carry out planning, motion control, and estimation in sequence over a sliding horizon.

Contrary to infotaxis, wherein agent is limited to a set of predefined actions, FIM based approach chooses optimal actions (that satisfy the input constraints) by maximizing a scalar function of an analytically computed FIM over a sliding horizon. Inspired by this approach, the authors in $\left[26^{\bullet \bullet}\right.$ ] exploited this concept for the sensor model adapted from [47] and analytically derived the FIM associated to a pollutant source localization problem. Figure 4 shows an example of source localization and tracing using the new methodology proposed. The optimization problem consisted of finding a sequence of the agent's course angle that maximized a scalar valued function of the FIM, i.e. its determinant. Maximizing the information about the source location resulted in steering the searcher towards the source. One can balance between gathering new knowledge versus exploiting existing knowledge by choosing the sliding time horizon. The FIM approach could possibly suffer from overestimation or underestimation as stated by [54 ${ }^{\bullet}$ ] for infotaxis. To the best of our knowledge, the successful implementation of the FIM method was reported for the first time in [53], where the objective is to localize a moving underwater target using a surface vehicle that measures its range to the target.

\section{Discussion}

The source localization problem has attracted enormous interest over the years and many methods have been proposed with various degrees of sophistication and complexity. In this article, we 


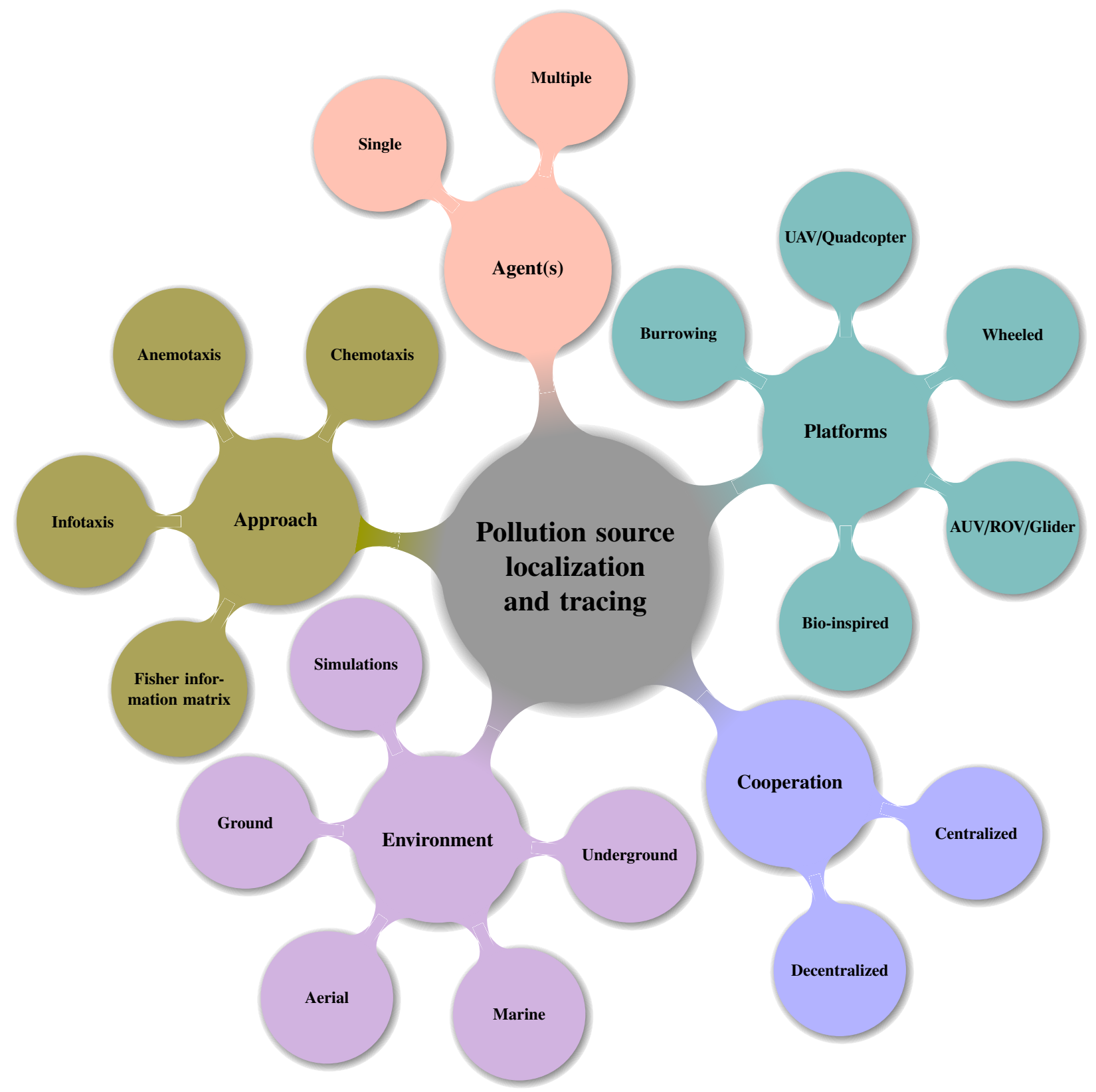

Figure 5 : Graphical abstract 
provided an overview of recent developments in this area that included various applications, different search methods, and platforms employed. Aerial applications have been investigated very extensively due to their early development, ease of deployment, and the use of wind tunnels to simulate an odor source [14]. In contrast to aerial applications, the marine technology is relatively new and still facing challenges such as long sensor response time, low bandwidth underwater communications, and limited vehicle capabilities for cruising and hovering.

Among the search methods, chemotaxis and anemotaxis are model-independent, which is a drawback if the medium is turbulent $\left[15^{\circ}\right]$. The latter causes disconnected patches of chemical substances with low or high dose, thus making it more difficult to get a good gradient estimation of the scalar field. Moreover, in chemotaxis or anemotaxis there is no feedback mechanism to minimize the uncertainty in the source location. Information based approaches, FIM and infotaxis, use a model of the pollutant in turbulent medium to minimize the uncertainty about the source location. This may lead to poor performance if there is a severe model mismatch [54 $\left.{ }^{\bullet}\right]$. It is worth mentioning that foraging strategies in nature may exhibit differently, such as the one reported in [56], which showed that mice can efficiently track odor sources with limited prior experience of source locations and consistent with a gradient ascent algorithm. Although, with more experience, they shifted from a sensory driven approach to a more efficient and stereotyped foraging approach that varied little in response to sensory data. Interestingly in [26 $\left.{ }^{\bullet}\right]$ one can notice that the optimal trajectory obtained has a close resemblance with the moth trajectory as reported in [56].

To summarize, we have provided the state-of-art in the domain of various search methods using different search platforms for source localization, as shown graphically in Figure 5. Although there is less freedom to pick a platform, there are mainly four methods, namely chemotaxis, anemotaxis, infotaxis, and FIM, depending on the application, and each of these methods has its own advantages and disadvantages. The gradient method is simple to implement compared to others but can be troublesome in the presence of turbulences. However, turbulences can be more effectively dealt by using the infotaxis method, which is a gradient-free method. From a conceptual point of view, information maximization seems to be very promising and may prove to play a significant role in many applications in the near future. Finally, we discuss future directions that warrant some attention.

In contrast to single-agent approaches that may require the agent to undergo complicated motions and may take a larger time to converge, multi-agent systems with complementary sensor suites offer a better way to approximate the gradient of signal measured by individual agents and shared among the agents in a centralized or distributed fashion. This helps to drive the formation of agents towards the source $\left[10^{\bullet}\right],[12,23,35,42,50,51,52,57,58,59,60]$. Since multi-agent systems provide flexibility, maneuverability to explore regions, and more information, we envision that they hold a great potential and will likely dominate future research directions in the source localization problem. However, from a theoretical standpoint this requires solution to a much harder problem but there are still challenges that needs to be addressed such as testing in open environments, hardware implementation and system integration, and cooperative localization and tracing. 


\section{References}

In what follows, papers published within the period of review (last five years) that are of special and outstanding interests are designated by $[\bullet]$ and $[\bullet \bullet]$, respectively.

[1] M. Dunbabin, L. Marques, Robots for environmental monitoring: Significant advancements and applications, IEEE Robotics and Automation Magazine 19 (1) (2012) 24-39. doi:10.1109/MRA.2011.2181683.

[2] A. M. Hein, F. Carrara, D. R. Brumley, R. Stocker, S. A. Levin, Natural search algorithms as a bridge between organisms, evolution, and ecology, Proceedings of the National Academy of Sciences 113 (34) (2016) 94139420. doi:10.1073/pnas.1606195113.

[3] G. Kowadlo, R. a. Russell, Robot Odor Localization: A Taxonomy and Survey, The International Journal of Robotics Research 27 (8) (2008) 869-894. doi:10.1177/0278364908095118.

[4] T. O. Fossum, A review of AUV autonomy and data-driven sample strategies, Tech. rep., Norwegian University of Science and Technology (2016).

[5] T. S. Moore, K. M. Mullaugh, R. R. Holyoke, A. S. Madison, M. Yücel, G. W. Luther, Marine chemical technology and sensors for marine waters: Potentials and limits, Annual Review of Marine Science 1 (1) (2009) 91-115. doi:10.1146/annurev.marine.010908.163817.

[6] S. Sankaran, L. R. Khot, S. Panigrahi, Biology and applications of olfactory sensing system: A review, Sensors and Actuators B: Chemical 171-172 (2012) 1-17. doi:10.1016/j.snb.2012.03.029.

[7] R. A. Russell, Comparing search algorithms for robotic underground chemical source location, Autonomous Robots 38 (1) (2015) 49-63. doi:10.1007/s10514-014-9396-x.

[8] P. P. Neumann, V. Hernandez Bennetts, A. J. Lilienthal, M. Bartholmai, J. H. Schiller, Gas source localization with a micro-drone using bio-inspired and particle filter-based algorithms, Advanced Robotics 27 (9) (2013) 725-738. doi:10.1080/01691864.2013.779052.

[9] B. Smith, M. Beman, D. Gravano, Y. Chen, Development and validation of a microbe detecting UAV payload, in: Workshop on Research, Education and Development of Unmanned Aerial Systems (RED-UAS), IEEE, 2015, pp. 258-264. doi:10.1109/RED-UAS.2015.7441015.

[10]• J. M. Soares, A. P. Aguiar, A. M. Pascoal, A. Martinoli, A distributed formation-based odor source localization algorithm - design, implementation, and wind tunnel evaluation, in: IEEE International Conference on Robotics and Automation (ICRA), IEEE, 2015, pp. 1830-1836. doi:10.1109/ICRA.2015.7139436.

A range-based formation control scheme was employed for odor source localization and tracing in a $2 D$ wind tunnel via Laplacian feedback formation control of multiple cooperative robots in a variety of formations. Source localization was done by plume centering of formation based on odor measurements obtained from all the robots in the formation.

[11] J. M. Soares, A. P. Aguiar, A. M. Pascoal, A. Martinoli, A graph-based formation algorithm for odor plume tracing, in: Springer Tracts in Advanced Robotics, Vol. 112, Springer, 2016, pp. 255-269. doi:10.1007/978-4431-55879-8_18.

[12] J. M. Soares, A. Marjovi, J. Giezendanner, A. Kodiyan, A. P. Aguiar, M. Pascoal, A. Martinoli, Towards 3-D distributed odor source localization : An extended graph-based formation control algorithm for plume tracking, in: IEEE/RSJ International Conference on Intelligent Robots and Systems (IROS), Daejeon, Korea, 2016, pp. $1729-1736$.

[13] R. A. Russell, A. Bab-Hadiashar, R. L. Shepherd, G. G. Wallace, A comparison of reactive robot chemotaxis algorithms, Robotics and Autonomous Systems 45 (2) (2003) 83-97.

[14] A. Marjovi, L. Marques, Optimal swarm formation for odor plume finding, IEEE Transactions on Cybernetics 44 (12) (2014) 2302-2315. doi:10.1109/TCYB.2014.2306291.

[15]• N. Voges, A. Chaffiol, P. Lucas, D. Martinez, Reactive searching and infotaxis in odor source localization, PLoS Computational Biology 10 (10) (2014) 1-13. doi:10.1371/journal.pcbi.1003861.

Inspired by moths, the authors attached an electroantennogram of a moth to a robot to indirectly control it. They compared four search strategies, three reactive (combination of upwind surge, spiral casting and crosswind [zigzag] casting), and infotaxis. The key conclusion was that the reactive search methods are more efficient (yielding shorter trajectories) for higher pheromone doses, while infotaxis performed better under lower doses of pheromones but with a more computational cost. 
[16]• S. M. Petillo, H. Schmidt, Autonomous and adaptive underwater plume detection and tracking with AUVs: Concepts, methods, and available technology, in: IFAC Conference on Manoeuvring and Control of Marine Craft (MCMC), IFAC, Arenzano (GE), Italy, 2012, pp. 232-237. doi:10.3182/20120919-3-IT-2046.00040.

This article provides a comparative study of using underwater gliders, AUVs, and remotely operated vehicles (ROVs) for plume detection and tracking. The highlight of this work was that actively propelled AUVs are the most well suited platform for such tasks due to their autonomy and sufficient payloads, despite their shorter endurance.

[17] W. Li, J. A. Farrell, S. Pang, R. M. Arrieta, Moth-inspired chemical plume tracing on an autonomous underwater vehicle, IEEE Transactions on Robotics 22 (2) (2006) 292-307.

[18] Yu Tian, Wei Li, Aiqun Zhang, Jiancheng Yu, Qifeng Zhang, Yan Li, From simulation to validation: Mothinspired chemical plume tracing with an autonomous underwater vehicle, in: IEEE Oceans Conference, 2014, pp. 1-10. doi:10.1109/OCEANS.2014.7002997.

[19] J. Das, F. Py, J. B. J. Harvey, J. P. Ryan, A. Gellene, R. Graham, D. A. Caron, K. Rajan, G. S. Sukhatme, Data-driven robotic sampling for marine ecosystem monitoring, The International Journal of Robotics Research 34 (12) (2015) 1435-1452. doi:10.1177/0278364915587723.

[20] M. Ohashi, Y. Kagawa, T. Nakatsuka, H. Ishida, Crayfish robot that generates flow field to enhance chemical reception, Journal of Sensor Technology 02 (04) (2012) 185-195. doi:10.4236/jst.2012.24026.

[21] B. Bayat, A. Crespi, A. Ijspeert, Envirobot: A bio-inspired environmental monitoring platform, in: IEEE/OES Conference on Autonomous Underwater Vehicles (AUV), Tokyo, Japan, 2016, pp. 1-6.

[22] X. Tan, Autonomous robotic fish as mobile sensor platforms: Challenges and potential solutions, Marine Technology Society Journal 45 (4) (2011) 31-40. doi:10.4031/mtsj.45.4.2.

[23] R. Cui, Y. Li, W. Yan, Mutual information-based multi-AUV path planning for scalar field sampling using multidimensional RRT*, IEEE Transactions on Systems, Man, and Cybernetics: Systems 46 (7) (2016) 9931004. doi:10.1109/TSMC.2015.2500027.

[24] F. Zhang, O. Ennasr, E. Litchman, X. Tan, Autonomous sampling of water columns using gliding robotic fish: Algorithms and harmful-algae-sampling experiments, IEEE Systems Journal 10 (3) (2016) 1271-1281. doi:10.1109/JSYST.2015.2458173.

[25] Y. Wang, R. Tan, G. Xing, J. Wang, X. Tan, Profiling aquatic diffusion process using robotic sensor networks, IEEE Transactions on Mobile Computing 13 (4) (2014) 880-893. doi:10.1109/TMC.2013.18.

[26]•• B. Bayat, N. Crasta, H. Li, A. Ijspeert, Optimal search strategies for pollutant source localization, in: IEEE/RSJ International Conference on Intelligent Robots and Systems (IROS), Daejeon, Korea, 2016, pp. 1-7.

This recent article was aimed at exploring the trajectories of a search agent (equipped with a sensor) that maximizes a scalar function of an appropriately defined Fisher information matrix with pollutant source localization as an unknown parameter. The optimization problem consisted of finding a sequence of the agent's course angle that maximized a scalar valued function of the FIM, i.e. the determinant of FIM. Maximizing the information about the source location resulted in steering the searcher towards the source. One can balance between gathering new knowledge versus exploiting existing knowledge by choosing the sliding time horizon.

[27] L. Champagne, E. Carl, R. Hill, Search theory, agent-based simulation, and u-boats in the Bay of Biscay, in: Proceedings of the 2003 International Conference on Machine Learning and Cybernetics, 2003, pp. 991-998. doi:10.1109/WSC.2003.1261521.

[28] K. B. Haley, L. D. Stone (Eds.), Search Theory and Applications, NATO Advanced Research Institute on Search Theory and Applications, Praia Da Rocha, Portugal, 1979. doi:10.1007/978-1-4615-9188-7.

[29] C. Paliotta, D. J. Belleter, K. Y. Pettersen, Adaptive source seeking with leader-follower formation control, in: IFAC Conference on Manoeuvring and Control of Marine Craft (MCMC), Elsevier B.V., Copenhagen, Denmark, 2015, pp. 285-290. doi:10.1016/j.ifacol.2015.10.294.

[30] G. Drews, Contributions of theodor wilhelm engelmann on phototaxis, chemotaxis, and photosynthesis, Photosynthesis Research 83 (1) (2005) 25-34. doi:10.1007/s11120-004-6313-8.

[31] V. Braitenberg, Vehicles: Experiments in synthetic psychology, MIT press, 1986.

[32] A. S. Matveev, M. C. Hoy, A. V. Savkin, 3D environmental extremum seeking navigation of a nonholonomic mobile robot, Automatica 50 (7) (2014) 1802-1815. doi:10.1016/j.automatica.2014.05.014.

[33] A. S. Matveev, M. C. Hoy, A. V. Savkin, Extremum seeking navigation without derivative estimation of a mobile 
robot in a dynamic environmental field, IEEE Transactions on Control Systems Technology 24 (3) (2016) 10841091. arXiv:1502.02224, doi:10.1109/TCST.2015.2467207.

[34] • B. Ristic, A. Skvortsov, A. Gunatilaka, A study of cognitive strategies for an autonomous search, Information Fusion 28 (2016) 1-9. doi:10.1016/j.inffus.2015.06.008.

One of the most recent articles on using infotaxis as a tool for pollution localization and tracing which is based on theoretical information principles to maximize the entropy reduction. It covers a short survey of the works done using infotaxis. The main contributions of proposed infotaxis based method are extending the estimation parameter space to include the source strength and implementing the search algorithm in the sequential Monte Carlo framework as a particle filter.

[35] Y.-X. Wu, Q.-H. Meng, Y. Zhang, M. Zeng, A novel chemical plume tracing method using a mobile sensor network without anemometers, in: Mechanical Engineering and Technology, Springer, 2012, pp. $155-162$. doi:10.1007/978-3-642-27329-2_22.

[36] D. Chang, W. Wu, D. R. Webster, M. J. Weissburg, F. Zhang, A bio-inspired plume tracking algorithm for mobile sensing swarms in turbulent flow, IEEE International Conference on Robotics and Automation (ICRA) (2013) 921-926.

[37] X. Zhang, Y. Sun, J. Xiao, F. Cabrera-Mora, Theseus gradient guide: An indoor transmitter searching approach using received signal strength, in: IEEE International Conference on Robotics and Automation (ICRA), Shanghai, China, 2011, pp. 2560-2565. doi:10.1109/ICRA.2011.5980454.

[38] J. S. Kennedy, Zigzagging and casting as a programmed response to wind-borne odour: A review, Physiological Entomology 8 (2) (1983) 109-120. doi:10.1111/j.1365-3032.1983.tb00340.x.

[39] A. Mafra-Neto, R. T. Cardé, Fine-scale structure of pheromone plumes modulates upwind orientation of flying moths, Nature 369 (6476) (1994) 142-144. doi:10.1038/369142a0.

[40] K. McCarthy, Cover story: REMUS-A role model for AUV technology transfer, International Ocean Systems 7 (6) (2003) 22-23.

[41] Y. Tian, W. Li, F. Zhang, Moth-inspired plume tracing via autonomous underwater vehicle with only a pair of separated chemical sensors, in: IEEE/MTS Oceans Conference, Washington DC, USA, 2015, pp. 1-8.

[42] X. Kang, W. Li, Moth-inspired plume tracing via multiple autonomous vehicles under formation control, Adaptive Behavior 20 (2) (2012) 131-142. doi:10.1177/1059712311433131.

[43] G. Lafferriere, J. Caughman, A. Williams, Graph theoretic methods in the stability of vehicle formations, in: American Control Conference (ACC), 2004, pp. 3729-3734.

[44] J. M. Soares, A. P. Aguiar, A. M. Pascoal, A. Martinoli, An algorithm for formation-based chemical plume tracing using robotic marine vehicles, in: MTS/IEEE Oceans Conference, Monterey, CA, 2016. doi:10.1109/oceans.2016.7761119.

[45] P. C. Abreu, J. Botelho, P. Gois, A. Pascoal, J. Ribeiro, M. Ribeiro, M. Rufino, L. Sebastiao, H. Silva, The MEDUSA class of autonomous marine vehicles and their role in EU projects, in: MTS/IEEE Oceans Conference, Shanghai, China, 2016, pp. 1-10. doi:10.1109/OCEANSAP.2016.7485620.

[46] Y. Zhang, X. Ma, Y. Miao, Multiple chemical sources localization using virtual physics-based robots with release strategy, Mathematical Problems in Engineering (2015) 1-16.

[47] M. Vergassola, E. Villermaux, B. I. Shraiman, Infotaxis as a strategy for searching without gradients, Nature 445 (7126) (2007) 406-409. doi:10.1038/nature05464.

[48] D. Martinez, E. M. Moraud, Reactive and cognitive search strategies for olfactory robots, CRC Press, 2013, Ch. 5, pp. 153-172.

[49] A. Eggels, R. Kunnen, B. Koren, A. Tijsseling, Infotaxis in a turbulent 3D channel flow, Journal of Computational and Applied Mathematics 310 (2017) 44-58.

[50] H. Hajieghrary, M. A. Hsieh, I. B. Schwartz, Multi-agent search for source localization in a turbulent medium, Physics Letters A 380 (20) (2016) 1698-1705. doi:10.1016/j.physleta.2016.03.013.

[51] H. Hajieghrary, A. F. Tomas, M. A. Hsieh, An information theoretic source seeking strategy for plume tracking in 3D turbulent fields, in: IEEE International Symposium on Safety, Security, and Rescue Robotics (SSRR), 2016, pp. 1-8.

[52] N. Fatès, Collective infotaxis with reactive amoebae: A note on a simple bio-inspired mechanism, in: J. Wa̧s, G. C. Sirakoulis, S. Bandini (Eds.), Lecture Notes in Computer Science, Vol. 8751, Springer International Pub- 
lishing, Cham, 2016, pp. 157-165. doi:10.1007/978-3-319-44365-2_15.

[53] N. Crasta, D. Moreno-Salinas, B. Bayat, A. M. Pascoal, J. Aranda, Optimal motion planning algorithm using range-only information in the presence of unknown currents, in: IFAC Conference on Control Applications in Marine Systems (CAMS), Trondheim, Norway, 2016, pp. 1-5.

[54]• J. D. Rodríguez, D. Gómez-Ullate, C. Mejía-Monasterio, Limits on the performance of infotaxis under inaccurate modelling of the environment, CoRR abs/1408.1 (2014) 1-8.

This interesting reference points out the limitations of the informations based methods such as infotaxis and FIM. These methods remain robust as long as the estimated parameters of a source are within a neighborhood of their true values. If an agent severely underestimates or overestimates the real environment parameters, the success rate quickly drops, making the search no longer feasible.

[55] D. Moreno-Salinas, N. Crasta, M. Ribeiro, B. Bayat, A. M. Pascoal, J. Aranda, Integrated motion planning, control, and estimation for range-based marine vehicle positioning and target localization, in: IFAC Conference on Control Applications in Marine Systems (CAMS), Trondheim, Norway, 2016, p. 6.

[56] A. Mafra-Neto, R. T. Cardé, Fine-scale structure of pheromone plumes modulates upwind orientation of flying moths, Nature 369 (1994) 142-144.

[57] J. Han, Y. Chen, Multiple UAV formations for cooperative source seeking and contour mapping of a radiative signal field, Journal of Intelligent and Robotic Systems: Theory and Applications 74 (1-2) (2014) 323-332. doi:10.1007/s10846-013-9897-4.

[58] M. B. Nogueira, J. Sousa, F. L. Pereira, Underwater plume tracing with an AUV cooperative navigation scheme based on the simplex algorithm, in: Mediterranean Conference on Control and Automation, 2013, pp. 251-256. doi:10.1109/MED.2013.6608730.

[59] D. Oswald, H. Lin, X. Mao, W. Li, L. Niu, X. Chen, A fleet of chemical plume tracers with the distributed architecture built upon DaNI robots, in: Advances in Swarm and Computational Intelligence, Vol. 9142, Springer, 2015, pp. 535-542. doi:10.1007/978-3-319-20466-6_56.

[60] L. Briñón-Arranz, L. Schenato, Consensus-based source-seeking with a circular formation of agents, in: European Control Conference (ECC), Zurich, Switzerland, 2013, pp. 2831-2836. 